\title{
Contribution of Visual I nput to the Subjective Visual Vertical During Prolonged Lateral Tilt Position
}

\author{
Fumiyuki Goto ${ }^{1,4 *}$, Tomoko Tsutumi ${ }^{1}$, Hironari Kobayashi ${ }^{2}$, Akira Saito $^{3}$, Jin Kanzaki ${ }^{4}$, Takanobu Kunihiro ${ }^{4}$ and Kaoru Ogawa ${ }^{4}$
}

${ }^{1}$ Department of Otorhinolaryngology Hino Municipal Hospital, Japan

${ }^{2}$ Department of Otorhinolaryngology, Saiseikai Chuo Hospital, Japan

${ }^{3}$ Department of Otorhinolaryngology, Saitama Chuou Hospital, Japan

${ }^{4}$ Department of Otorhinolaryngology, Keio University, School of Medicine, Japan

\begin{abstract}
Objective: The aim of this study was to investigate changes of the subjective visual vertical (SVV) in healthy, male subjects during prolonged lateral body tilt both in light and dark conditions.
\end{abstract}

Methods: Static SVVs were obtained in a sitting and in lying down positions. Following the measurement in the sitting position, the subject was instructed to lay down and remain that way for the next set of recordings. SVV was measured immediately and repeated another 8 times at 15-minute intervals. Finally, the subject returned to the sitting position, and SVV was measured immediately and 15 minutes later, both in light and dark conditions.

Results: Tilt of the SVV shifted in the same direction as the head tilt soon after the subject assumed the lateral body tilts position from the sitting position. This SVV deviation gradually increased, eventually reaching a plateau. Although subjects reached the plateau at various rates, the mean latency to reach the plateau were approximately 30 minutes. This phenomenon was reproduced with intra-subject repeated testing. SVV deviation increased more rapidly in the light than in the dark.

Conclusion: Comparison of SVVs under dark and light conditions informs us that the phenomenon, which is the subjective recognition of contralateral tilt of the true vertical, could function in the maintenance of one's head in the vertical position. We propose this visual effect is one of important inputs to stabilize visual vertical. Therefore visual input would be essential for maintaining one's upright position on earth.

Keywords: Vestibular; Subjective visual vertical; Otolith; Ocular counter-rolling

\section{Introduction}

The subjective perception of contralateral tilt of the subjective vertical while in the supine position was first reported by Aubert and is termed the A-phenomenon (Aubert's phenomenon) [1]. At slight body tilt angles, the true vertical appears to shift towards the same direction as the body tilt (i.e. ipsilateral side) and is termed the $\mathrm{E}$ -phenomenon (Mueller phenomenon) [2]. Both phenomena have been studied extensively [3-5]. However, changes in the SVV under different visual conditions of prolonged lateral body tilt positions have not yet been investigated. We sometimes feel sense of unsteadiness after awakening following a night's sleep. We hypothesize that this unsteadiness is related to temporal changes in graviceptive function that occur when the body is positioned horizontally for prolonged periods. As a first step in testing this hypothesis, we investigated the contribution of visual inputs to temporal changes in SVV when subjects change from a sitting to a prolonged lying position.

\section{Methods}

We examined 11 healthy, male subjects (age range: 30 to 41 years; mean age \pm s.d.: $36.5 \pm 0.4$ years). None of the subjects had a history of peripheral or central vestibular disorders, and their neuroophthalmological and neuro-otological examinations were normal. All subjects gave their informed consent to participate in the study according to the guidelines of the ethics committee of Keio University Hospital. SVV was measured as a psycho physiological variable for perceived verticality in a static condition, as in our previous reports [6,7].

Subjects gazed binocularly into a hemispherical dome $(60 \mathrm{~cm}$ in diameter), the inner surface of which was covered with a random dot pattern (Figure 1). The dome was deprived of horizontal or vertical cues. A small, round, clear plastic board $(10 \mathrm{~cm}$ in diameter), on which an illuminated bar $(1 \mathrm{~cm} \times 10 \mathrm{~cm})$ was projected, was attached to the center of the rotating dome. The subject could adjust the illuminated bar to his subjective vertical using a potentiometer. The SVV was

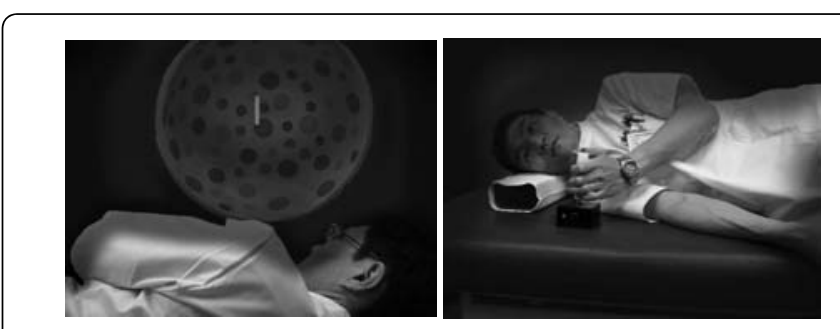

Figure 1: The photograph of recording in lay down condition. Subjects gazed binocularly into a hemispherical dome $(60 \mathrm{~cm}$ in diameter). A small, round, clear plastic board (10 cm in diameter), on which an illuminated bar $(1 \mathrm{~cm} \times 10$ $\mathrm{cm}$ ) was attached to the center of the rotating dome.

*Corresponding author: Fumiyuki Goto M.D. Ph.D, Department of Otorhinolaryngology, Hino Municipal Hospital, Tamadaira 4-3-1, Hino-shi, Tokyo 191-0062, Japan,Tel: +81-42-581-2677; Fax: +81-42-581-2923; E-mail: amifumi@ bc5.so-net.ne.jp

Received August 19, 2010; Accepted October 19, 2010; Published October 21 2010

Citation: Goto F, Tsutumi T, Kobayashi H, Saito A, Kanzaki J, et al. (2010) Contribution of Visual Input to the Subjective Visual Vertical during Prolonged Lateral Tilt Position. J Neurol Neurophysiol 1:103. doi:10.4172/2155-9562.1000103

Copyright: (c) 2010 Goto F, et al. This is an open-access article distributed unde the terms of the Creative Commons Attribution License, which permits unrestricted use, distribution, and reproduction in any medium, provided the original author and source are credited. 
taken as the average of 10 consecutive adjustments of the illuminated bar from a random offset position to the subjective vertical when the dome was stationary. The SVV was denoted as positive when the tilt was clockwise (CW) and negative when counterclockwise (CCW) from the subject. Statistical analysis was performed with

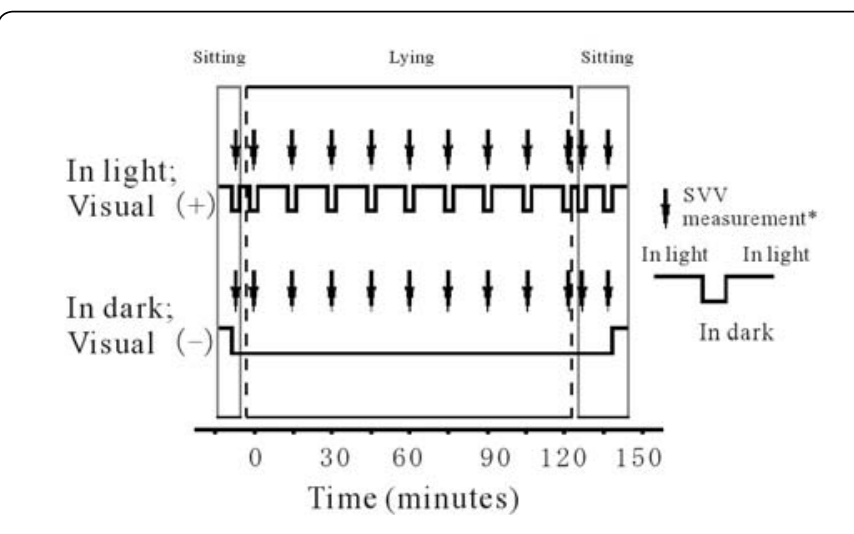

Figure 2: Schematic representation of the experimental protocol. SVV measurements were taken in subjects in sitting and lying positions. Measurements were carried out under light and dark conditions. *SVV measurements were repeated 10 times at 5-10second intervals.

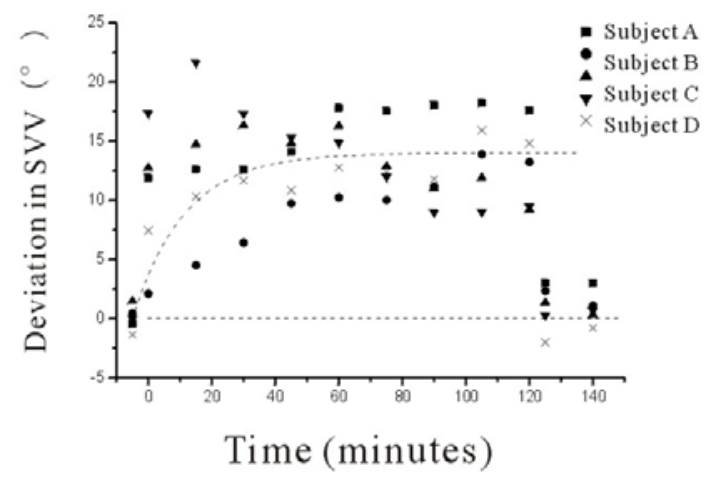

Figure 3: Temporal changes in SVV measured during lighted conditions in subjects lying down on their left sides. Data from 5 representative subjects are shown. SVV deviated almost immediately after the subjects laid down, plateauing in about 30 minutes.

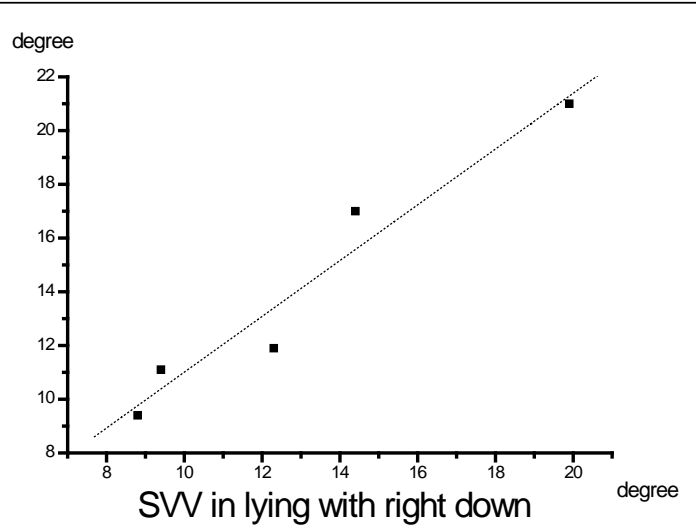

Figure 4: Comparison of SVVs measured from subjects lying down on their left- versus right-sides. There is statistically significant correlation between them $(R=0.97, P<0.01)$. commercially available software (Microcal Origin; Northampton, MA). ANOVA was employed for statistical comparisons. Exponential fitting of the temporal changes in SVV was performed according to the least squares method.

\section{Experiment 1}

All measurements were collected under both light and dark conditions (Figure 2). For the light condition, the subject assumed a sitting position and was instructed to view the surroundings, the light was turned off, and an SVV measurement was taken. For the dark condition, the room remained dark for the whole session, and the testing procedures were as follows. The initial SVV was measured with the subject in a sitting position, and his head was unrestrained. The second measurement was taken soon after the subject was instructed to lay down on his left side on a platform, his head resting on a pillow (Figure 1). The height of the pillow was adjusted so that an imaginary line bisecting the subject's pupils was perpendicular to the platform (i.e., earth horizontal). Measurements were repeated 9 times at 15-minute intervals. The subject returned to the sitting position, and 15 minutes later a final measurement was taken. In 5 subjects, measurements were repeated 3 times on different days to confirm the reproducibility of our findings. After adequate rest, the same recording procedure was performed while subjects were lying down on their right sides. These measurements were carried out only under lighted conditions.

\section{Experiment 2}

To assess temporal changes in SVV more precisely, SVV was measured continuously for 10 minutes in 5 subjects soon after they assumed the lying position without any experimenter offset. The moving average was calculated every 10 seconds for quantitative comparison. The same kind of continuous recordings were carried out 3 times, every 2 weeks to verify reproducibility of our results.

\section{Results}

Temporal changes in SVV measured in subjects in the lying down position. Both the deviation and dispersion of the SVV measured from subjects in the sitting Position was quite small. In contrast, the deviation and dispersion of SVV measured from subjects lying down on their left sides were comparatively larger. Moreover, as soon as the subject lay down, the SVV immediately deviated toward the same direction as the subjects' heads. Over time, deviation of the SVV increased exponentially, reaching a plateau in 30 minutes. Representative data from 5 subjects are shown in (Figure 2). Similar results were obtained when the subjects were lying down on their right sides. Comparison of SVVs measured from subjects lying down on their left- versus right-sides. There is no significant difference between sides $(n=5)$.

\section{Contribution of visual input}

Under both light and dark conditions, SVV tilt rapidly increased soon after the subject assumed a lying down position, then it plateaued (Figure 5). The SVV recorded in the sitting position was statistically different from the first SVV measurement recorded in the lying position. The first and second measurements recorded in the lying position in the dark were also statistically different. The first and second SVV measurements recorded in the lying position in the light were not statistically different. This indicates that lying down for 15 minutes with no ambient visual stimulation was insufficient for the SVVs to reach a plateau; 30 minutes was required. There were no statistical differences between plateau SVV values measured in 


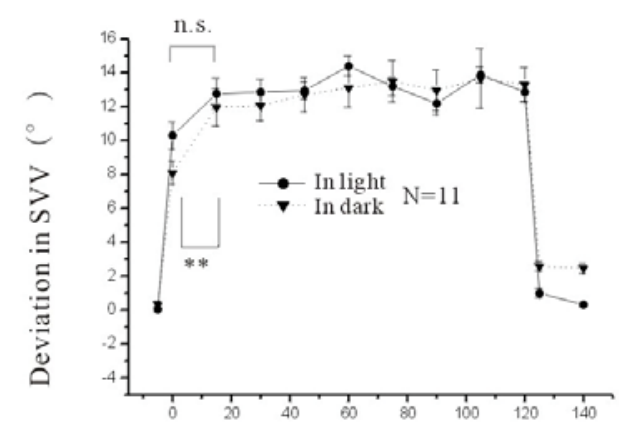

Time (minutes)

$$
\text { **: } p<0.01 \text {, n.s.: not significant. }
$$

Figure 5: Contribution of visual input to temporal changes in SVV measured in subjects lying down. All subjects were lying on their left sides during SVV measurements. Error bars represent standard error of means. Although the first and second SVV measurements recorded in the lying position (i.e. 15-minute interval between sitting and lying) in the light were not statistically different, the first and second measurements recorded in the lying position in the dark were statistically different.

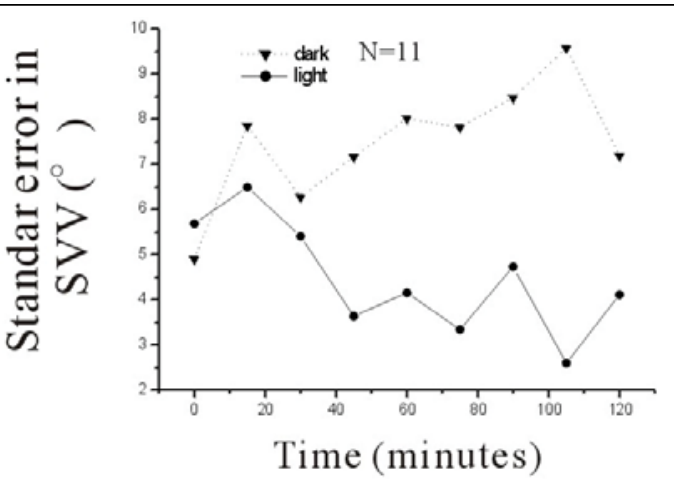

Figure 6: Changes in standard error of SVVs measured during light and dark conditions. Data are from subjects lying with their left sides down.

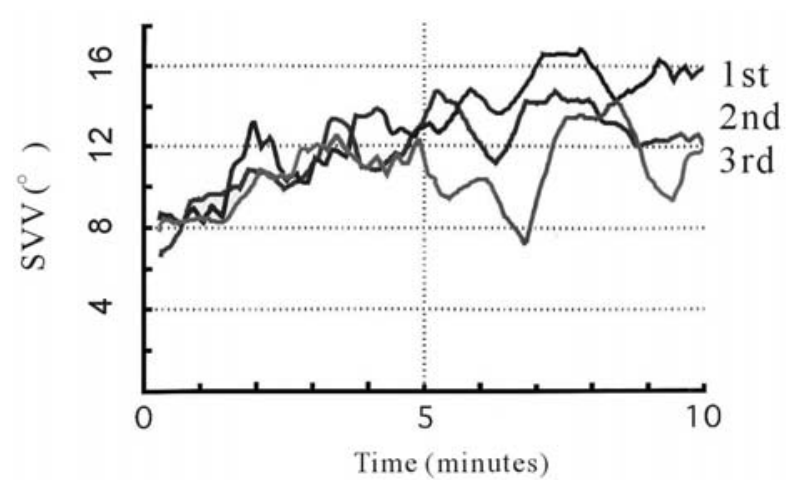

Figure 7: Temporal changes in mean SVV measured during dark conditions. Moving average was calculated from SVVs measured for 10 seconds continuously. Recordings were repeated following at least an hour rest.

light and dark conditions. The dispersion of SVVs measured at each time point was usually smaller in experiments carried out under light conditions compared to that of experiments carried out under dark conditions (Figure 6).

\section{Continuous recording}

Reproducibility of SVV results was examined (Figure 7). The dispersion of SVVs decreased over time in light; however, SVV deviation increased exponentially, as in (Figure 3). A similar trend was observed in all subjects.

\section{Discussion}

There is significant role played by proprioceptive and contact cues in the perception of verticality while lying down. In experiments with patients, tilts of perceived vertical (SVV) is known as a sign of a vestibular tone imbalance in roll. A lesion induced vestibular tone imbalance should result in a syndrome consisting of a perceptual tilt (SVV), head and body tilt. In general tilt of SVV is accompanied with ocular tilt reaction (OTR). Clinical signs of lesions attributed to graviceptive pathways manifest in this reaction [8]. The OTR is an oculocephalic response consisting of ipsilateral head tilt, skew deviation, and ocular torsion (OT) [9]. OT and OCR are used synonymously. A close relationship between SVV and OT has been reported [10]. Therefore, SVV tilt can be interpreted as the result of an OCR to the subject assuming a lying position. Of course, for this to be true, the contribution of other sensory input, such as proprioceptive and visual stimuli, should be negligible.

In our experiments, deviation of SVV increased immediately after the subject laid down. SVV is affected not only by OCRs related to otolith inputs, but also by other sensory inputs. The decreased dispersion of SVVs observed during light conditions indicates that visual inputs significantly contribute to SVV. When a subject rotates his head, the resulting OCR is an OT or eye rotation in the direction opposite to that of head rotation [11]. This response is mediated by the otolith organs, especially the utricle [12]. Many factors (e.g., state of arousal, visual stimulation) influence this phenomenon [13-15]. During extended periods of lateral body tilt, temporal changes in OCR, in terms of torsional angle, varied in different subjects. The OCRs of some subjects plateaued, whereas the OCRs of others decreased over time. In contrast, temporal changes in SVV were similar in all subjects. Although we did not investigate the relationship between OCR and SVV, this apparent disparity in temporal changes can be explained by how OCRs and SVVs were measured. OCRs were measured in the dark, with the subjects' eyes open, whereas SVVs were only measured in the light, with the subjects' eyes open.

Dispersion of SVVs measured during lighted conditions decreased with time, suggesting that visual input stabilizes the body for SVV determination. Different visual conditions do not affect the maximum SVV. Wade [16] described the contribution of various postural systems to directional responses to prolonged body tilts. They mentioned the large contribution of the trunk system to visual orientation. Our findings clarify the importance of visual input to the visual vertical. The A-phenomenon plays a role in enhancing graviceptive pathways via visual input. This is essential for keeping the head in a vertical position.

\section{Conclusions}

We investigated the temporal changes in SVV in subjects laying in a prolonged lateral position. Soon after assuming the lying position, the SVV of all subjects tilted toward the same direction as their head tilt. This response, the A-phenomenon, progressively increased before reaching a plateau. Visual input shortened the time required to reach the plateau. Once the plateau was reached, SVVs measured with and without visual input were the same. Dispersion of SVVs measured in the presence of visual input decreased with time, 
Citation: Goto F, Tsutumi T, Kobayashi H, Saito A, Kanzaki J, et al. (2010) Contribution of Visual Input to the Subjective Visual Vertical during Prolonged Lateral Tilt Position. J Neurol Neurophysiol 1:103. doi:10.4172/2155-9562.1000103

Page 4 of 4

whereas dispersion of SVVs measured in the absence of visual input was unchanged. These results indicate that visual input stabilizes the body for SVV determination. The A-phenomenon plays a role in enhancing graviceptive pathways via visual input. This is important for keeping the head vertical.

\section{Acknowledgement}

This research was supported by a Grant-in-Aid from Keio University for the Encouragement of Young Medical Scientists (01-0207).

\section{References}

1. von Aubert $\mathrm{H}$ (1861) Eine scheinbare bedoutende Drehung von Objecten bei Neigung des Kopfes nach rechts oder links. Arch Pathol Anat 20: 381-393.

2. Mueller C, Kornilova L, Wiest $G$, Deecke $L$ (1994) Visually induced vertical selfmotion Sensation is altered in microgravity. J Vestib Res 4: 161-167.

3. Van Beuzekom AD, Medendorp WP, Van Gisbergen JA (2001) The subjective vertical and the sense of self orientation during active body tilt. Vision Res 41 : 3229-3242.

4. Van Beuzekom AD, Van Gisbergen JA (2000) Properties of the internal representation of gravity inferred from spatial-direction and body-tilt estimates. J Neurophysiol 84: 11-27.

5. Curthoys IS (1996) The role of ocular torsion in visual measures of vestibular function. Brain Res Bull 40: 399-403.

6. Goto F, Kobayashi H, Hayashi Y, Higashino K, Kunihiro T, et al. (2003)
Compensatory changes in static and dynamic subjective visual vertical in patients following vestibular schwanoma surgery. Auris Nasus Larynx 30: 2933.

7. Kobayashi H, Hayashi Y, Higashino K, Saito A, Kunihiro T, et al.(2002) Dynamic and static subjective visual vertical with aging. Auris Nasus Larynx 29: 325-328.

8. Vibert D, Hausler R (2003) Acute peripheral vestibular deficits after whiplash injuries. Ann Otol Rhinol Laryngol 112: 246-51.

9. Brandt T (1999) Vestibular disordes in (frontal) roll plane. In: Brandt T, editor. Vertigo: Its multisensory syndromes. 2 ndedn London: Springer

10. Dieterich M, Brandt T (1993) Ocular torsion and tilt of subjective vetrtical are sensitive brainstem signs. Ann Neurol 33: 292-299.

11. MILLER EF 2nd (1962) Counterrolling of the human eyes produced by head tilt with respect to gravity. Acta OtoLaryngol 54: 479-501.

12. Groen E, Bos JE, Nacken PF, de Graaf B (1996) Determination of ocular torsion by means of automatic pattern recognition. IEEE Trans Biomed Eng 43: 471479.

13. Kobayashi N, Yashiro T, Ishii M, Sekiguchi C (2001) Factors influencing ocular counterrolling. Oto-rhino-laryngology, Tokyo 44: 457-65.

14. Kobayashi N (1998) Relationship between changes in OCR and directional cognition inlong term tilt loading. Equilibrium Res 57: 522-528.

15. Nomura Y, Watanabe $Y$, Igarashi M, Sudoh M, Sekiguchi C, et al. (2000) Ocular counter-rolling after prolonged alteration in the direction of gravity. Nippon Jibiinkoka Gakkai Kaiho 103: 916-921.

16. Wade SW, Curthoys IS (1997) The effect of ocular torsional position on perception of the roll-tilt of visual stimuli. Vision Res 37: 1071-1078. 